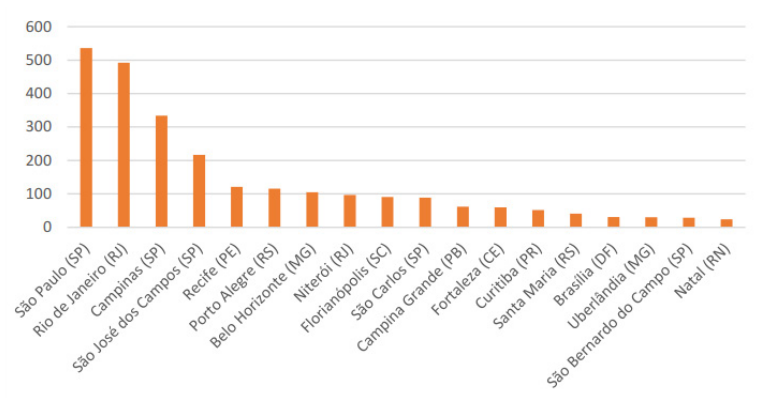

*Possui graduação em Arquitetura e Urbanismo pela Universidade do Vale do Rio dos Sinos (1987), mestrado em Arquitetura e Urbanismo pela Universidade de São Paulo (1996) e doutorado em Arquitetura e Urbanismo pela Universidade de São Paulo (2003).

usjt • arq.urb • número 24 | janeiro - abril de 2019 em Arquitetura e Urba da Universidade Federal do Espírito Santo desde 2017

\title{
A relação entre as produções científicas e o desenvolvimento das Cidades Inteligentes brasileiras
}

The relation between the scientific productions and the development of the Brazilian Smart Cities

Cristina Engel de Alvarez* e Lívia Campos Salzani**

\section{Resumo}

As questões relacionadas às Smart Cities (Cidades Inteligentes) têm sido um tema recorrente, seja no âmbito dos poderes públicos municipais, seja no meio acadêmico através de publicações científicas. Nesse sentido, o Brasil também vem ampliando a discussão sobre o tema, com ampla - e, muitas vezes, indevida - divulgação nas mídias sobre algum "ranking" de inteligência alcançado por determinada cidade, obtido através de indicadores nacionais ou internacionais. O objetivo desta pesquisa foi quantificar as publicações do cenário acadêmico brasileiro sobre as cidades inteligentes e aferir se algumas das cidades brasileiras consideradas inteligentes possuem alguma correspondência entre sua produção científica e sua colocação no ranking Connected Smart Cities, feito pela empresa Urban Systems. Para isso, foi selecionada uma plataforma de base de dados de pesquisas científicas - a Engineering Village, pertencente à editora internacional Elsevier - para realizar um levantamento bibliométrico e relacionar a quantidade de produções científicas das cidades, tendo por recorte temporal o período de 1990 a 2017. A partir do ranking escolhido, foi analisado se aquelas cidades com maiores produções científicas foram consideradas, efetivamente, as mais inteligentes. Como resultado, identificou-se que oito das dez cidades mais bem classificadas no ranking Connected Smart Cities apresentam um número relevante de publicações.

Palavras-chave: Rankings. Cidades Inteligentes. Smart Cities. Periódico acadêmico.

\section{Abstract}

The questions surrounding Smart Cities have been an ongoing theme, be it on city halls and the public sphere, be it on Academia through scientific publications. Following this trend, Brazil has also been broadening the discussion surrounding this theme, with a wide - and, many times, improper - divulge in the media about some kind of "ranking" of intelligence being reached by a certain city, achieved through national or international indicators. The goal of this research was to quantify publications in the Brazilian academic context about smart cities assess if any of the Brazilian cities ranked as smart actually match their scientific production to their position on the ranking Connected Smart Cities, made by Urban Systems. In order to do that a scientific research data base platform was selected Engineering Village, it belongs to Elsevier an international publisher- to make a bibliometric mapping and relate the amount of scientific productions of the cities, using the time period of 1990 a 2017 as base. Based on the chosen ranking, it was analyzed that if the cities with the largest scientific productions were considered, effectively, the smartest. As a result, it was noted that eight out of the ten cities ranked the best in the ranking Connected Smart Cities have a relevant member of publications.

Keywords: Rankings. Smart Cities. Academic papers. 


\section{Introdução}

Com o rápido crescimento da população mundial e migração para as cidades a partir da segunda metade do século passado, cerca de $54 \%$ da população mundial vive em meios urbanos, sendo que, no Brasil, esse número já ultrapassou $85 \%$ da população (ONU, 2014). A falta de planejamento gerou problemas em relação à infraestrutura, utilização eficiente dos recursos naturais e descarte de resíduos, qualidade do ar, desemprego, educação, insalubridade das moradias, etc., criando uma urgência na busca de estratégias mais inteligentes para acompanhar o crescimento e minimizar ou resolver os problemas criados (NAM; PARDO, 2011; ANTHOPOULOS; VAKALI, 2012; DINIZ et al., 2015).

No final da década de 1980, quando a sustentabilidade do meio urbano foi amplamente discutida, e com a popularização da internet a partir da década de 1990, iniciaram-se os primeiros estudos e discussões a respeito dos avanços tecnológicos e suas possíveis aplicações ao planejamento urbano e gerenciamento das cidades, o que culminou na formulação do termo Smart Cities. Inicialmente, o termo esteve associado a uma forma de visualizar o contexto urbano aliado às novas tecnologias da informação e comunicação, utilizando-as para melhorar os serviços públicos, diagnósticos de problemas e compartilhamento de informações (ANGELIDOU, 2015).

$\mathrm{Na}$ bibliografia consultada para o referencial teórico desta pesquisa, observa-se que não há um consenso em relação ao conceito Smart Cities, ou Cidades Inteligentes, no entanto, adotou-se o entendimento de serem cidades que utilizam as Tecnologias de Informação e Comunicação (TIC's) em associação à criatividade em relação ao capital humano, cooperação entre as partes interessadas e ideias científicas para implementar melhorias na infraestrutura municipal e promover facilidades para seus habitantes (GIFFINGER et 
al., 2007; LOMBARDI, 2011; CARAGLIU; DEL BO, 2011; ANTHOPOULOS; VAKALI, 2012; ANGELIDOU, 2014).

Contudo, em países da Europa, América do Norte e também da América Latina e Brasil, o uso de TIC'S já está inserido no cotidiano de um grande número de cidades, em pequena ou grande escala, explorado por entidades públicas e privadas, seja nas áreas de saúde, engenharia, produção, abastecimento de água, transporte público, gestão de resíduos, manufatura ou finanças, em que os sistemas de informação se integram para aproximar usuários de serviços públicos, prover sistemas mais eficientes, diagnosticar falhas, aumentar capacidades ou facilitar pagamentos e financiamentos (PUPO, 2017).

Foram vários os estudos que, na busca pela conceituação do termo "Smart Cities", criaram indicadores e categorias de avaliação, sendo, em sua maioria, coincidentes nos seguintes principais temas: governança, mobilidade, economia, ambiente, vivências e pessoas. Alguns autores citam, ainda, que devem ser levados em consideração mais aspectos, tais como recursos naturais e energia, construções (NEIROTTI et al. 2014), tecnologia (CHOURABI et al., 2012; ANGELIDOU, 2015), condições de saúde, espírito inovador, produção de resíduos (LAZAROIU; ROSCIA, 2012) entre outros.

É fundamental, também, estabelecer a diferenciação entre as Cidades Inteligentes, Cidades
Digitais e Cidades Sustentáveis. A Cidade Digital é aquela que prioriza o uso das tecnologias de comunicação em seus sistemas para melhorar os serviços públicos de forma a atender melhor os servidores, cidadãos e as organizações. Já as Cidades Sustentáveis são caracterizadas por se voltarem para soluções voltadas não só para problemas relacionados ao meio ambiente, mas também econômicos e sociais, sem os quais a verdadeira sustentabilidade não é possível. Já as Cidades Inteligentes emergem das Cidades Digitais, também abraçando alguns aspectos das Cidades Sustentáveis, porém tendo o capital humano e social como foco de seus esforços (WEISS; BERNARDES; CONSONI, 2015; AHVENNIEMI et al., 2017).

Ainda sobre os termos "smart" ou "inteligente", apesar de serem aqueles utilizados no meio acadêmico e na mídia, ainda levantam questionamentos uma vez que as cidades avaliadas têm consideráveis diferenças culturais, geográficas e econômicas, sendo a comparação entre elas uma tarefa difícil e pouco eficiente. O estudo desenvolvido por PRZEYBILOVICZ et al. (2018), comparou duas cidades brasileiras e suas soluções, a fim de analisar a influência dos diferentes usos das TIC's: a primeira fazia amplo uso de tecnologia para melhorar eficiência e performance de serviços públicos, atribuindo-lhes maior qualidade, enquanto a segunda, apesar de não possuir um projeto voltado para o amplo uso das tecnologias, possuía um funcionamento eficiente e tinha um foco maior em transparência política 


\begin{tabular}{|c|c|}
\hline Autores & Definição \\
\hline $\begin{array}{l}\text { NAM; PARDO } \\
(2011 \text {, p. } 284)\end{array}$ & $\begin{array}{l}\text { "Uma cidade mais inteligente infunde informações em sua estrutura física para aprimorar } \\
\text { conveniências, facilitar mobilidade, contribuir para eficiências, conservar energia, melhorar } \\
\text { a qualidade do ar e da água, identificar problemas e conserta-los rapidamente, se recuperar } \\
\text { rapidamente de desastres, coletar dados para tomar melhores decisões, utilizar recursos } \\
\text { com eficiência e compartilhar dados para permitir a colaboração entre as entidades e do- } \\
\text { mínios." }\end{array}$ \\
\hline $\begin{array}{l}\text { CARAGLIU; DEL BO; } \\
\text { NIJKAMP (2011, p. 70) }\end{array}$ & $\begin{array}{l}\text { "Nós acreditamos uma cidade ser inteligente quando investimentos em capital humano e } \\
\text { social e tradicional (transporte) e infraestrutura de comunicação moderna (TIC), combustí- } \\
\text { veis sustentáveis, crescimento econômico e alta qualidade de vida, com um gerenciamento } \\
\text { sensato dos recursos naturais através de participação no governo." }\end{array}$ \\
\hline $\begin{array}{l}\text { A N THOP O ULOS; } \\
\text { VAKALI }(2012, \text { p. 178) }\end{array}$ & $\begin{array}{l}\text { "Hoje elas aprimoram conteúdo digital e serviços nas áreas urbanas, incorporam computa- } \\
\text { ção disseminada e enfrentam desafios ambientais. Vários casos internacionais apresentam } \\
\text { abordagens alternativas para a cidade inteligente, enquanto capitalizam as Tecnologias de } \\
\text { Informação e Comunicação (TIC's) para múltiplos propósitos, que variam de serviços sim- } \\
\text { ples de entregas a coleta de dados sofisticadas para tomadas de decisões municipais." }\end{array}$ \\
\hline $\begin{array}{l}\text { NEIROTTI; et al } \\
(2014, \text { p. } 26)\end{array}$ & $\begin{array}{l}\text { "Basicamente, as várias posições no debate concordam com o fato de que uma Cidade } \\
\text { Inteligente deve ser capaz de otimizar o uso e exploração de ambos ativos, tangíveis (in- } \\
\text { fraestruturas de transporte, redes de distribuição de energia, recursos naturais) e intangí- } \\
\text { veis (capital humano, capital intelectual de companhias e organização do capital na admi- } \\
\text { nistração pública)." }\end{array}$ \\
\hline $\begin{array}{l}\text { WEISS; BERNARDES; } \\
\text { CONSONI }(2015, \text { p. 2) }\end{array}$ & $\begin{array}{l}\text { "Ela (a Cidade Inteligente) utiliza as capacidades da cidade digital para implementar siste- } \\
\text { mas de informações que melhorem a disponibilidade e a qualidade das infraestruturas e } \\
\text { serviços públicos, incrementando sua capacidade de crescimento e estimulando a inova- } \\
\text { ção e o desenvolvimento sustentável." }\end{array}$ \\
\hline $\begin{array}{l}\text { CÂMARA } \\
(2016, \text { p. 139) }\end{array}$ & $\begin{array}{l}\text { "Nesse caso, o conceito de inteligente se refere à condição de uma população ativa, com- } \\
\text { prometida com o interesse coletivo, motivada a participar, identificando os problemas e se } \\
\text { sentindo capaz de contribuir para as soluções." }\end{array}$ \\
\hline $\begin{array}{l}\text { MAZZO } \\
(2018, \text { p.36) }\end{array}$ & $\begin{array}{l}\text { "Uma cidade inteligente promove as pessoas no centro do desenvolvimento, absorve tec- } \\
\text { nologias de informação e comunicação em sua gestão e consegue sincronizar esses ele- } \\
\text { mentos como ferramentas que promovem a formação de um governo eficiente, o qual } \\
\text { apresenta um planejamento colaborativo e uma participação ativa da sociedade. Smart Ci- } \\
\text { ties favorecem esse desenvolvimento integrado e sustentável, tornando-se mais eficientes, } \\
\text { competitivas e inovadores, aumentando a qualidade de vida de sua população." }\end{array}$ \\
\hline $\begin{array}{l}\text { GUIMARÃES } \\
(2018, \text { p. 121) }\end{array}$ & $\begin{array}{l}\text { "Cidade inteligente é uma cidade que tem o cidadão empoderado como protagonista e } \\
\text { beneficiário de suas ações e as tecnologias de informação e comunicação como coadju- } \\
\text { vantes principais e meios habilitadores para uma gestão pública transparente, participativa, } \\
\text { responsiva e efetiva. É uma cidade que se renova e inova de maneira integrada, sistêmica e } \\
\text { sistemática, na busca do bem comum da sociedade e de suas futuras gerações." }\end{array}$ \\
\hline
\end{tabular}

Figura 1. Definição das Cidades Inteligentes de acordo com os autores selecionados. e incentivo à participação popular nos processos de tomadas de decisões. Os autores concluem que as diferentes formas de se aplicar as TIC's em projetos de Smart Cities ainda desperta tensão e controvérsia ao se analisar a iniciativa como inteligente ou não e sugerem, dessa forma, que a "inteligência" das cidades não tem que estar, necessariamente, vinculada às questões tecnológicas ou relacionadas ao uso das TICs no cotidiano dos cidadãos.

A tabela da Figura 1 apresenta as definições do termo "cidade inteligente" de acordo com os autores estudados no referencial bibliográfico, no entanto, destaca-se que não é objeto deste estudo a discussão sobre a relação entre o que se denomina como "cidade inteligente" e a qualidade de vida de seus usuários, e tampouco serão discutidos o efetivo significado do termo e a natural polêmica sobre a eventual associação entre "inteligência" e "automação".

Como as Cidades Inteligentes possuem desdobramentos complexos, compreendendo características socioambientais, tecnológicas, econômicas, administrativas e urbanísticas, o assunto é pesquisado por diversas áreas do conhecimento, com cada pesquisador ou grupo de pesquisa conferindo seu foco, como planejamento urbano (NEIROTTI et al., 2014), economia urbana (CARAGLIU; DEL BO; NIJKAMP, 2012), sistemas de informação (CHOURABI et al., 2012) etc. A proposta deste trabalho foi identificar as principais instituições brasileiras que têm publicado sobre

usjt • arq.urb • número 24 | janeiro - abril de 2019 
o tema a fim de relacionar a produção científica brasileira e as cidades com melhores classificações no ranking Connected Smart Cities.

\section{Rankings de Cidades Inteligentes}

Para alguns pesquisadores acadêmicos, os rankings não possuem valor científico, uma vez que são realizados por empresas privadas com fins de lucro próprio e cujas metodologias não são divulgadas, de forma que não podem ser replicadas. Porém, a competitividade entre cidades para serem mais inteligentes têm utilizado essas ferramentas como uma forma para se autopromoverem, a fim de atrairrm profissionais qualificados, empreendedores e fomentar atividades turísticas (GUIMARÃES; PLONSKI, 2018). Assim, os rankings de classificação são um dos meios de comparação mais utilizados para demonstrar quais são aquelas com as melhores características e potenciais para determinadas atividades e investimentos através de uma pontuação (GIFFINGER et al., 2007; ROCHE, 2012).

Apesar de a maioria dos processos de avaliação e respectivos indicadores não apresentarem uma metodologia clara que possa ser replicada, bem como verifique-se a resistência plenamente justificada de alguns pesquisadores em relação ao uso dos rankings de classificação, a decisão de adoção dos mesmos como objeto deste estudo foi justamente no intuito de verificar se há uma efetiva correspondência entre os mesmos e os estudos desenvolvidos. É preocupante o fato de que os rankings sejam mecanismo que possuem ampla divulgação na mídia e, consequentemente, uma maior visibilidade do público, tanto leigo quanto de possíveis empreendedores.

Algumas iniciativas foram tomadas para se definir indicadores que determinem se uma cidade pode ser classificada como inteligente ou não. Tais indicadores, normalmente mensuráveis, buscam avaliar através de um processo comparativo, a partir da adoção de marcas de referência, que podem ou não ser utilizadas também para ponderar as melhorias que vão sendo alcançadas com o tempo. Assim, de acordo com os defensores desse processo, pode-se então produzir dados que permitam uma análise comparativa entre cidades, ou mesmo a definição de parâmetros de referência que sejam guias orientativos para buscar soluções cada vez mais inteligentes.

Na Europa, foi criado pela Technische Universitat Wien o ranking European Smart Cities que, desde 2007, vem estudando as cidades inteligentes de tamanho médio da Europa, tendo publicado classificações destas em 2007, 2013 e 2014 e uma para grandes cidades em 2015 (TECHNISCHE UNIVERSITAT WIEN, 2015). De acordo com a classificação do ranking, a cidade de Luxemburgo foi classificada em 2007 em primeiro lugar, passando ao segundo na avaliação de 2013 e voltando ao primeiro em 2014, como pode ser visto nas imagens da Figuras 2, 3 e 4, cada uma com os primeiros 10 colocados no ranking dos anos de 2007, 2013 e 2014, respectivamente. (Figura 2, 3 e 4). 


\begin{tabular}{|c|c|c|c|c|c|c|c|}
\hline Stadt & $\underline{E c o}$ & $\underline{\text { Peo }}$ & $\underline{\text { Gov }}$ & Mob & Env & $\underline{\text { Liv }}$ & Total \\
\hline LU Luxembourg & 1 & 2 & 13 & 6 & 25 & 6 & 1 \\
\hline DK Aarhus & 4 & 1 & 6 & 9 & 20 & 12 & 2 \\
\hline Fl Turku & 16 & 8 & 2 & 21 & 11 & 9 & 3 \\
\hline DK Aalborg & 17 & 4 & 4 & 11 & 26 & 11 & 4 \\
\hline DK Odense & 15 & 3 & 5 & 5 & 50 & 17 & 5 \\
\hline Fl Tampere & 29 & 7 & 1 & 27 & 12 & 8 & 6 \\
\hline Fl Oulu & 25 & 6 & 3 & 28 & 14 & 19 & 7 \\
\hline NL Eindhoven & 6 & 13 & 18 & 2 & 39 & 18 & 8 \\
\hline AT Linz & 5 & 25 & 11 & 14 & 28 & 7 & 9 \\
\hline AT Salzburg & 27 & 30 & 8 & 15 & 29 & 1 & 10 \\
\hline FR Montpellier & 30 & 23 & 33 & 24 & 1 & 16 & 11 \\
\hline
\end{tabular}

Figura 2. As 10 cidades melhores colocadas no ranking European Smart Cities publicado em 2007. Siglas: Áustria (AT), Dinamarca (DK), Finlândia (FI), Holanda (NL) e Luxemburgo (LU). Fonte: European Smart Cities, 2014. Acesso em 04 mar, 2019.

\begin{tabular}{|lcc|c|c|c|c|c|}
\hline \multicolumn{1}{l}{ City } & Eco & Peo & Gov & Mob & Env & Liv & Total \\
\hline DK AARHUS & 2 & 1 & 5 & 3 & 14 & 25 & 1 \\
\hline LU LUXEMBOURG & 1 & 15 & 50 & 4 & 22 & 4 & 2 \\
\hline DK AALBORG & 9 & 8 & 4 & 14 & 7 & 10 & 3 \\
\hline SE UMEAA & 21 & 5 & 1 & 31 & 2 & 11 & 4 \\
\hline FI TAMPERE & 19 & 2 & 11 & 30 & 1 & 22 & 5 \\
DK ODENSE & 12 & 6 & 3 & 21 & 11 & 35 & 6 \\
\hline SE JOENKOEPING & 29 & 10 & 2 & 11 & 6 & 20 & 7 \\
\hline AT SALZBURG & 25 & 18 & 25 & 2 & 23 & 1 & 8 \\
\hline SI LJUBLJANA & 4 & 3 & 31 & 25 & 18 & 21 & 9 \\
\hline AT LINZ & 11 & 20 & 29 & 8 & 24 & 6 & 10 \\
\hline AT INNSBRUCK & 31 & 23 & 23 & 13 & 12 & 3 & 11 \\
\hline
\end{tabular}

Figura 3. As 10 cidades melhores colocadas no ranking European Smart Cities 2.0 publicado em 2013. Siglas: Áustria (AT), Dinamarca (DK), Eslovênia (SI), Finlândia (FI), Luxemburgo (LU) e Suécia (SE). Fonte: European Smart Cities, 2013. Acesso em 


\begin{tabular}{|c|c|c|c|c|c|c|c|}
\hline City & Eco & Peo & Gov & Mob & Env & Liv & Total \\
\hline LU LUXEMBOURG & 1 & 18 & 56 & 4 & 16 & 4 & 1 \\
\hline DK AARHUS & 2 & 3 & 6 & 3 & 19 & 27 & 2 \\
\hline SE UMEAA & 24 & 5 & 2 & 34 & 1 & 13 & 3 \\
\hline SE ESKILSTUNA & 21 & 1 & 7 & 24 & 3 & 41 & 4 \\
\hline DK AALBORG & 10 & 11 & 5 & 14 & 14 & 10 & 5 \\
\hline SE JOENKOEPING & 32 & 13 & 3 & 11 & 2 & 26 & 6 \\
\hline DK ODENSE & 13 & 9 & 4 & 20 & 9 & 40 & 7 \\
\hline FI JYV̈̈SKYL̈̈ & 23 & 8 & 1 & 47 & 5 & 25 & 8 \\
\hline FI TAMPERE & 16 & 2 & 15 & 31 & 12 & 14 & 9 \\
\hline AT SALZBURG & 27 & 24 & 29 & 2 & 27 & 1 & 10 \\
\hline FI TURKU & 20 & 6 & 12 & 15 & 18 & 29 & 11 \\
\hline FI OULU & 14 & 4 & 9 & 39 & 13 & 35 & 12 \\
\hline AT INNSBRUCK & 35 & 27 & 26 & 12 & 6 & 3 & 13 \\
\hline AT LINZ & 11 & 23 & 31 & 8 & 25 & 7 & 14 \\
\hline SI LJUBLJANA & 6 & 7 & 34 & 33 & 21 & 21 & 15 \\
\hline
\end{tabular}

Figura 4. As 10 cidades melhores colocadas no ranking European Smart Cities 3.0 publicado em 2014. Siglas: Áustria (AT), Dinamarca (DK), Finlândia (Fl), Luxemburgo (LU) e Suécia (SE). Fonte: European Smart Cities, 2014. Acesso em 05 ago. 2017

No Brasil, a empresa Urban Systems realizou pela primeira vez em 2015 um ranking nacional de cidades inteligentes, o Connected Smart Cities, e o repetiu em 2016. Para a criação de seus indicadores, a empresa mapeou pesquisas, acadêmicas ou não, a fim de realizar o levantamento dos conceitos e viabilidade de cada indicador, porém a metodologia para a aplicação desses indicadores não se encontra disponível ao público (URBAN SYSTEMS, 2017). Os resultados são divulgados no site da empresa (fonte) e pela revista semanal Época (fonte).
No ano de 2016, mais de 500 cidades se submeteram à avaliação, porém, destas, apenas 100 foram classificadas. Foram considerados 70 indicadores divididos nos seguintes tópicos: mobilidade, urbanismo, meio ambiente, energia, tecnologia, educação, saúde, segurança, empreendedorismo, economia e governança. Parte do resultado do ranking pode ser visto nas $\mathrm{Fi}-$ guras 5 e 6 (URBAN SYSTEMS, 2017), em que também é apresentada uma comparação entre a classificação das cidades em relação à avaliação do ano anterior. 
Cristina Engel de Alvarez e Lívia Campos Salzani A relação entre as produções científicas e o desenvolvimento das Cidades Inteligentes brasileiras

\begin{tabular}{|c|c|l|r|}
\hline \multicolumn{2}{|c|}{ Posição } & \multicolumn{1}{|c|}{ Município (UF) } & Pontos \\
\hline 2017 & 2016 & & 33,197 \\
\hline 19 & 19 & São Paulo (SP) & 32,472 \\
\hline 29 & 39 & Curitiba (PR) & 32,125 \\
\hline 39 & 29 & Rio de Janeiro (RJ) & 30,785 \\
\hline 49 & 59 & Belo Horizonte (MG) & 30,426 \\
\hline 59 & 69 & Vitória (ES) & 30,281 \\
\hline 69 & 79 & Florianópolis (SC) & 29,987 \\
\hline 79 & 49 & Brasilia (DF) & 29,788 \\
\hline 89 & 109 & Campinas (SP) & 29,418 \\
\hline 99 & 139 & São Caetano do Sul (SP) & 29,339 \\
\hline 109 & 99 & Recife (PE) & \\
\hline
\end{tabular}

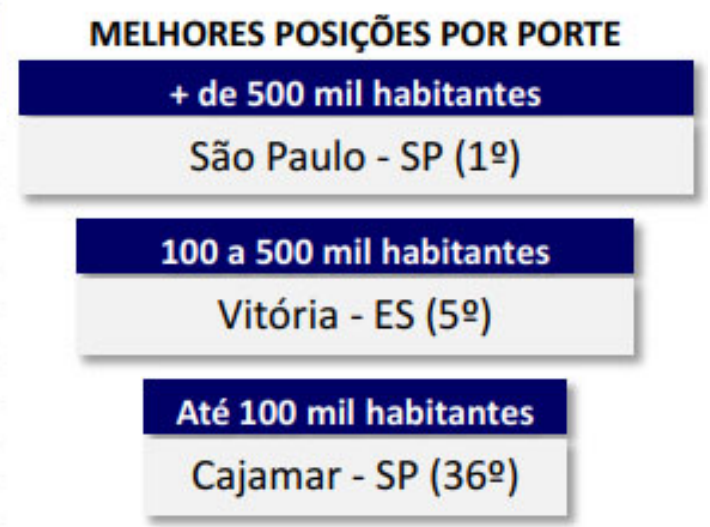

Figura 5. 10 cidades melhor classificadas no Ranking Connected Smart Cities. Fonte: adaptado de Urban Systems, 2017

\begin{tabular}{|c|c|c|c|c|c|c|c|}
\hline \multicolumn{2}{|c|}{ Posição } & \multirow{2}{*}{ Municipio (UF) } & \multirow{2}{*}{ Pontos } & \multicolumn{2}{|c|}{ Posição } & \multirow{2}{*}{ Municipio (UF) } & \multirow{2}{*}{ Pontos } \\
\hline 2017 & 2016 & & & 2017 & 2016 & & \\
\hline $11^{\circ}$ & $11^{\circ}$ & Porto Alegre (RS) & 29,283 & $31^{\circ}$ & $26^{\circ}$ & Piracicaba (SP) & 26,109 \\
\hline $12^{\circ}$ & $12^{\circ}$ & Santos (SP) & 29,026 & $32^{\circ}$ & $33^{\circ}$ & São José do Rio Preto (SP) & 25,944 \\
\hline $13^{\circ}$ & 89 & Barueri (SP) & 29,013 & $33^{\circ}$ & $59^{\circ}$ & Uberaba (MG) & 25,929 \\
\hline $14^{\circ}$ & $14^{9}$ & Campo Grande (MS) & 28,881 & $34 \%$ & $35^{\circ}$ & Itajai (SC) & 25,897 \\
\hline $15^{\circ}$ & $25^{\circ}$ & Palmas (TO) & 28,365 & $35^{\circ}$ & $27^{\circ}$ & Joinville (SC) & 25,737 \\
\hline $16^{\circ}$ & $15^{\circ}$ & Goiânia (GO) & 28,294 & $36^{\circ}$ & $67^{\circ}$ & Cajamar (SP) & 25,699 \\
\hline $17^{\circ}$ & $18^{\circ}$ & Salvador (BA) & 28,031 & $37^{\circ}$ & 249 & São José Dos Campos (SP) & 25,669 \\
\hline $18^{\circ}$ & $16^{\circ}$ & Niterói (RJ) & 27,894 & $38^{\circ}$ & 719 & Paulinia (SP) & 25,663 \\
\hline $19^{\circ}$ & $17^{\circ}$ & Maringá (PR) & 27,334 & $39 \%$ & 409 & Vinhedo (SP) & 25,657 \\
\hline $20^{\circ}$ & $30^{\circ}$ & São Bernardo do Campo (SP) & 26,978 & $40^{\circ}$ & $49^{\circ}$ & Resende (RJ) & 25,620 \\
\hline $21^{\circ}$ & $21^{\circ}$ & Jundiai (SP) & 26,968 & $41^{\circ}$ & $64^{\circ}$ & Colatina (ES) & 25,598 \\
\hline $22^{\circ}$ & $19^{\circ}$ & Ribeirão Preto (SP) & 26,956 & $42^{\circ}$ & 369 & Macaé (RJ) & 25,512 \\
\hline $23^{\circ}$ & $45^{\circ}$ & Londrina (PR) & 26,933 & $43^{\circ}$ & $38^{\circ}$ & Amparo (SP) & 25,438 \\
\hline $24^{\circ}$ & $32^{\circ}$ & Juiz de Fora (MG) & 26,730 & $44^{\circ}$ & $79^{\circ}$ & Cuiabá (MT) & 25,407 \\
\hline $25^{\circ}$ & $20^{\circ}$ & Petrópolis (RJ) & 26,569 & $45^{\circ}$ & $70^{\circ}$ & Limeira (SP) & 25,328 \\
\hline $26^{\circ}$ & $22^{\circ}$ & Santo André (SP) & 26,510 & $46^{\circ}$ & $28^{\circ}$ & Teresina (PI) & 25,208 \\
\hline $27^{\circ}$ & $23^{\circ}$ & Blumenau (SC) & 26,466 & $47^{\circ}$ & $47^{\circ}$ & Joāo Pessoa (PB) & 25,191 \\
\hline $28^{\circ}$ & $31^{\circ}$ & Uberlândia (MG) & 26,321 & $48^{\circ}$ & $83^{\circ}$ & Botucatu (SP) & 25,167 \\
\hline $29^{\circ}$ & $29^{\circ}$ & Fortaleza (CE) & 26,228 & 490 & $63^{\circ}$ & Presidente Prudente (SP) & 25,049 \\
\hline $30^{\circ}$ & $55^{\circ}$ & Indaiatuba (SP) & 26,146 & $50^{\circ}$ & $57^{\circ}$ & Marilia (SP) & 25,028 \\
\hline
\end{tabular}

Figura 6. cidades classificadas no Ranking Connected Smart Cities. Fonte: adaptado de Urban Systems, 2017. 
A principal questão que esta pesquisa buscou responder é se há uma equivalência entre a quantidade de publicações do conhecimento relacionado ao tema das cidades inteligentes e a classificação da cidade, ou seja, se o investimento feito para o desenvolvimento científico na área auxilia na efetiva replicabilidade no local de origem das pesquisas.

\section{Indicadores e iniciativas para cidades mais in- teligentes}

Os indicadores podem ser usados não somente para avaliar as cidades, mas também para sugerir as mudanças que podem ser inseridas para a implantação de infraestruturas e facilidades: "Indicadores são figuras ou medidas que habilitam informação sobre um fenômeno complexo, tal como impacto ambiental, para simplifica-lo em uma forma relativamente simples de usar e entender" (AHVENNIEMI et al., 2017, p. 236, tradução nossa).

Cada sistema de classificação tem sua maneira de propor indicadores. No estudo feito por Giffinger e Gudrun (2010), foram definidos 74 indicadores divididos dentro das 6 principais categorias. Porém, nos rankings Connected Smart Cities e European Smart Cities, esses números variam, provavelmente para melhor se adaptarem aos locais de estudo. O European Smart Cities, com colaboração de Giffinger, possui 81 indicadores, enquanto o brasileiro Connected Smart Cities conta com 70 indicadores. Os indicadores propostos por Giffinger e Gudrun (2010) seguem organizados de acordo com as principais características das cidades inteligentes:

- "Economia: espírito inovador, empreendedorismo, imagem econômica e marca comercial, produtividade, flexibilidade do mercado de trabalho, incorporação internacional, habilidade de transformar.

- Pessoas: nível de qualificação, afinidade com aprendizado a longo termo, pluralidade social e étnica, flexibilidade, criatividade, cosmopolitismo/receptividade, participação na vida pública.

- Governança: participação na tomada de decisões, serviço público e social, governo transparente, estratégias e perspectivas políticas.

- Mobilidade: acessibilidade local, acessibilidade internacional e nacional, disponibilidade de infraestrutura de TIC's, sistema de transportes sustentável, inovador e seguro.

- Ambiente: falta de poluição nas condições naturais, poluição, proteção ambiental, gerenciamento de recursos sustentáveis.

- Vivências: instalações culturais, condições de saúde, segurança individual, qualidade das habitações, instalações educacionais, turismo, coesão social” (GIFFINGER; GUDRUN, 2010, p. 14-15, tradução e adaptação nossa).

Apesar dos estudos sobre as Cidades Inteligentes, as diversas necessidades dos países impulsionam ações diferentes para a resolução de problemas, dificultando a identificação de tendências (NEIROTTI et al., 2014). Dentre algumas das ações das cidades para se tornarem mais inteligentes, pode-se tomar como exemplo a cidade de Luxemburgo, em primeiro lugar na últi- 
ma edição do ranking European Smart Cities para cidades de tamanho médio.

Entre algumas das suas iniciativas, estão o fornecimento de serviços digitais aos cidadãos, o acesso aos investimentos para inovações tecnológicas e os investimentos em ciclovias e calçadas mais acessíveis (LUXEMBOURG, 2017). Destaque é dado para os serviços Hot City, que fornecem internet gratuita na maior parte dos espaços públicos da cidade, e o $\mathrm{mLive}$, um projeto nacional de transporte público que ajuda o usuário a organizar seus trajetos via computador, tablet ou celular.

No Brasil, a campeã do ranking, São Paulo, destaca-se nas categorias de mobilidade, urbanismo, empreendedorismo, tecnologia e inovação. É a cidade brasileira com maior disponibilidade de modais, além de investir continuamente no melhoramento e expansão de suas linhas de metrô. De acordo com o Urban System (2016), suas novas leis de Plano Diretor e Uso e Ocupação do Solo possuem práticas inovadoras, em busca de uma cidade mais humana, moderna e equilibrada. São Paulo é reconhecida por ser uma cidade onde criam-se muitas pequenas empresas, inclusive algumas das quais fazem investimento em produção de novas tecnologias (URBAN SYSTEMS, 2016).

\section{Metodologia}

Para este trabalho foi realizada uma pesquisa quantitativa e exploratória baseada no trabalho publicado por Cruz et al. (2015) que escolheu uma plataforma de base de dados - a Engineering ViIlage - para avaliar como estavam sendo feitas as pesquisas sobre as Smart Cities pelo mundo, em que países predominavam as publicações, quais os principais autores, focos de pesquisa, entre outros aspectos. Nesse estudo foi concluído que o Brasil estava em $19^{\circ}$ lugar dos países com maior número de publicações sobre Smart Cities, sendo o único país da América Latina na listagem. Os autores concluíram que, de fato, existe uma preocupação da parte dos pesquisadores brasileiros em desenvolver mais estudos sobre as Cidades Inteligentes, passível de verificação através do crescimento em quantidade e qualidade das publicações ao longo dos anos.

Engineering Village é uma plataforma internacional, pertencente à editora Elsevier. A plataforma também foi escolhida para este estudo tanto pela respeitabilidade no meio científico como, também, por possibilitar a localização das publicações por país e afiliação dos autores. Assim, localizando-se a instituição onde as pesquisas foram desenvolvidas, pode-se localizar, também, as cidades onde foram produzidas.

Foram escolhidas apenas as publicações em periódicos, pois apesar de não representarem toda a pesquisa científica produzida, muitos são resultados de trabalhos maiores, provindos de teses 


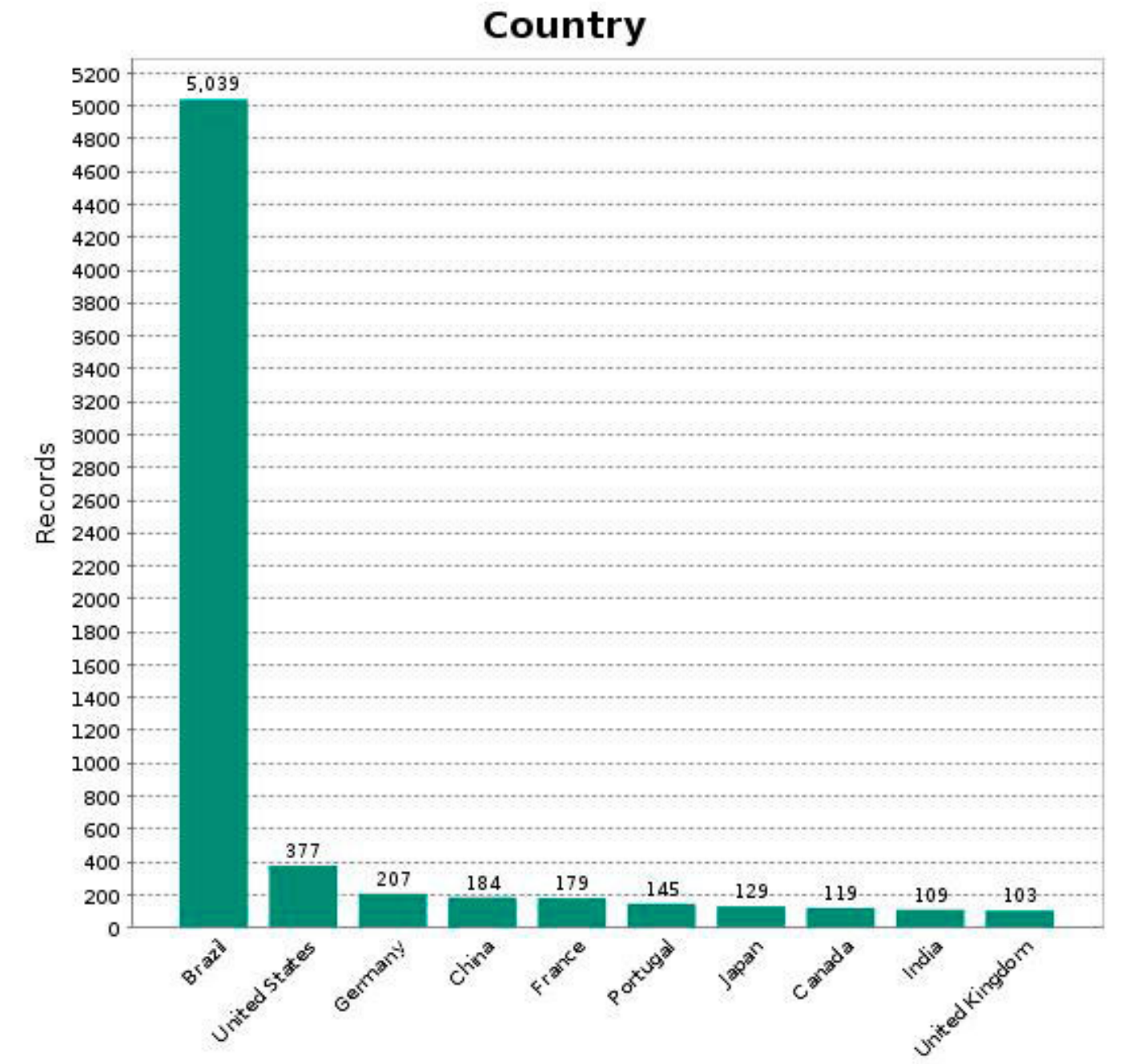

(9) 2017 Elsevier Inc.

Figura 7. Correspondência entre principais países de origem das pesquisas. Fonte: Engineering Village, 2017. e dissertações de pós-graduação. Além disso, teses e dissertações não passam por uma avaliação tão qualificada quanto a das pesquisas submetidas ao parecer para uma publicação em periódico. Dessa forma, quando esses resultados são publicados em periódicos revisados, são devidamente reconhecidos por sua qualidade e mérito.

Como palavras-chave para a pesquisa na plataforma, foi escolhida a combinação das palavras em inglês "'smart cities' OR 'smart city' AND Brazil OR brazilian'", de modo que fossem obtidas as produções relacionadas às cidades inteligentes referentes ao país, mesmo estas tendo sido publicadas em periódicos internacionais.

\section{Resultados}

Na pesquisa dos periódicos, realizada em setembro de 2017 pelas bases de dados na plataforma Engineering Village, foram encontrados 34.391 resultados. Desse número, 24.129 resultados são originários do país, havendo 32.761 resultados no idioma inglês e 1.235 em português. A plataforma fornece dados em formato de gráficos para melhor análise (Figura 7).

A partir das informações dos institutos de afiliação dos autores dos trabalhos científicos encontrados, pode-se localizar em que cidades essas pesquisas foram feitas. Comparando a lista das cidades com o ranking Connected Smart Cities, 
observa-se que o primeiro lugar, São Paulo, corresponde ao local com maior produção científica sobre cidades inteligentes no país, totalizando 536 pesquisas publicadas, enquanto o Rio de Janeiro, que ocupou a segunda posição em 2016 e que em 2017 está em terceiro, possui um total de 492 publicações. Campinas, que ficou com a $8^{a}$ posição, também se destaca com um número alto de publicações, 334, seguida por São José dos Campos, com 217. Apesar disso, São José dos Campos ficou com a $37^{\text {a }}$ colocação no ranking das cidades inteligentes.

Ainda que as demais cidades dentre as 10 mais inteligentes não tenham apresentado uma quan- tidade de publicações tão alta quanto a de São Paulo e Rio de Janeiro, também apresentam interesse pela temática das Cidades Inteligentes em suas publicações: Curitiba ( $2^{\mathrm{a}}$ colocada), 52 publicações; Belo Horizonte ( $4^{a}$ colocada), 105 publicações; Florianópolis (6a colocada), 91 publicações; Brasília ( $7^{a}$ colocada), 31 publicações e Recife $\left(10^{a}\right.$ colocada), 121 publicações. Apenas publicações originadas em Vitória ( $5^{a}$ colocada) e São Caetano do Sul ( $9^{a}$ colocada) não foram encontradas na pesquisa da base de dados. Dessa forma, tem-se que oito das dez primeiras colocadas apresentam uma relação entre produção científica e nível de inteligência. Uma análise da relação entre cidades e publicações é apresentada na Figura 8:

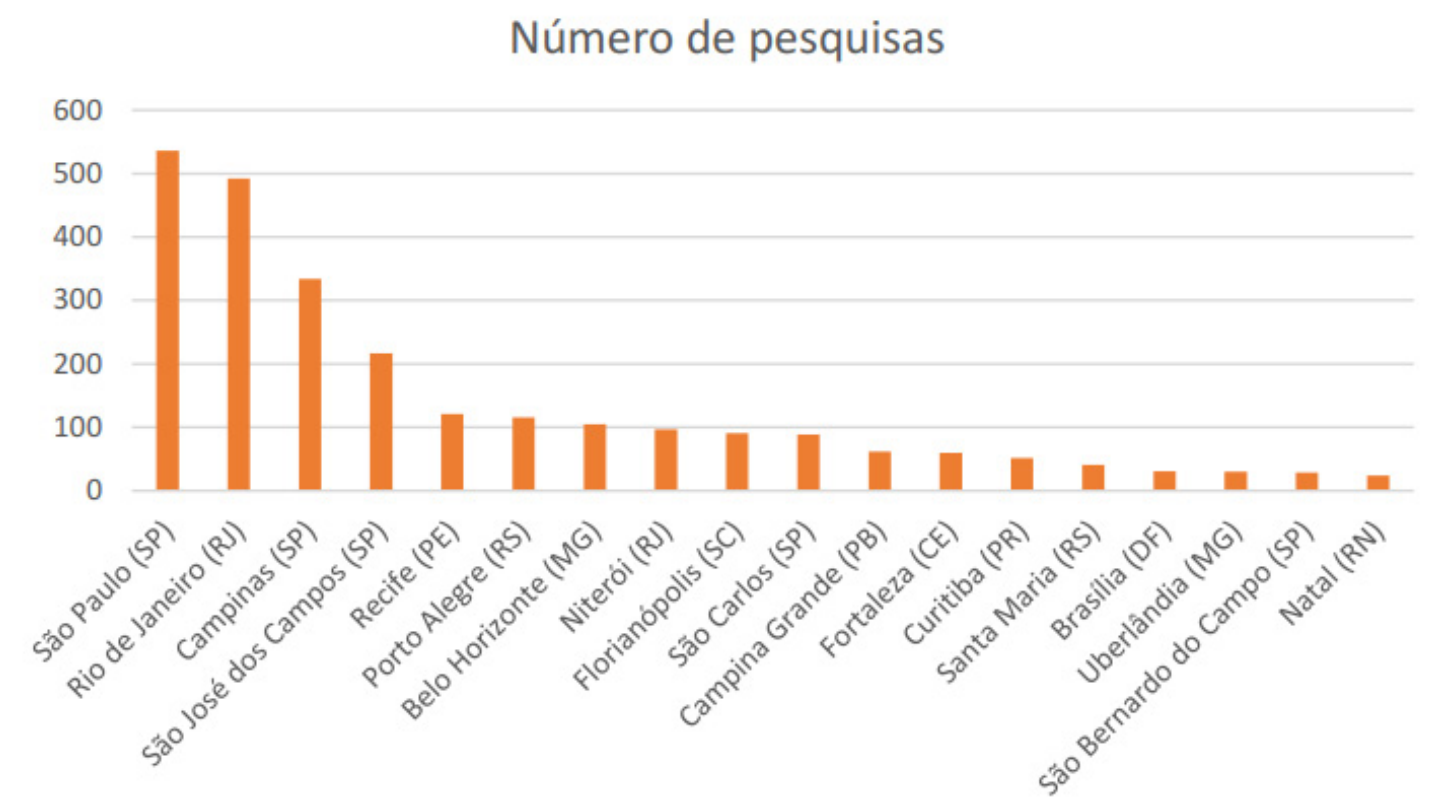

Figura 8. Quantitativo de publicações por cidades. Fonte: adaptado de Engineering Village. 


\section{Conclusões}

Este trabalho pode ser dividido em três partes onde primeiro se buscou contextualizar as definições de cidades inteligentes; em seguida foram apresentadas as classificações das cidades no ranking das cidades inteligentes e, por fim, foram comparadas as cidades com suas publicações científicas dentro do tema "Cidades Inteligentes", visando investigar a possível correlação entre desenvolvimento científico e a condição do lugar.

Conforme demonstrado anteriormente, oito das dez cidades mais bem classificadas apresentam um número considerável de publicações e quase todas as cidades com publicações encontradas estão dentro do ranking. Destaca-se também que a cidade classificada em primeiro lugar, São Paulo, também é aquela que possui um maior número de pesquisas publicadas relativas à temática. Dessa forma, pode-se afirmar que grande parte daquelas que estão mais bem posicionadas no ranking possuem uma produção acadêmica relevante acerca de Cidades Inteligentes e que isso pode estar influenciando diretamente no seu posicionamento.

Todavia, das 100 cidades selecionadas, apenas foram identificadas publicações relacionadas às universidades ou instituições de pesquisa de 18 cidades. Também foi visto que algumas cidades bem posicionadas - como Vitória ( $3^{\circ}$ lugar) e São Caetano do Sul ( $9^{\circ}$ lugar) -, não tiveram publicações identificadas no Engineering Village e outras, como Uberlândia $\left(28^{\circ}\right.$ colocada) e Fortaleza (29a colocada), foram identificadas publicações.

Os resultados encontrados podem levar a outras investigações mais especificas sobre essas cidades, acerca de como os poderes públicos destas avaliam as necessidades de mudanças fundamentais para que ocorram melhorias de maneira a se desenvolverem de forma mais inteligente. O Connected Smart Cities, dividindo o ranking por categorias, também apresenta as cidades que ficaram em primeiro lugar em cada uma delas. Sendo assim, outro desdobramento interessante seria avaliar a correlação das pesquisas existentes dentro de cada eixo temático com o desenvolvimento de tecnologias utilizadas para melhoramentos dos mesmos nas cidades.

Diante dos estudos realizados, pode-se inferir que a obtenção de uma cidade inteligente está relacionada, também, ao investimento acadêmico em pesquisas e na consequente publicação de alta qualidade. 


\section{Referências:}

AHVENNIEMI, Hannelle et al. What are the differences between sustainable and smart cities?. Cities, n. 60, p. 234-245, fev. 2017. Disponível em: <https://www.sciencedirect.com/science/ article/pii/S0264275116302578>. Acesso em: 11 mai. 2018.

ANGELIDOU, Margarita. Smart cities: a conjecture of four forces. Cities, n. 47: p. 95-106, set. 2015. Disponível em: <https://www.sciencedirect. com/science/article/pii/S0264275115000633>. Acesso em: 11 mai. 2018.

ANGELIDOU, Margarita. Smart cities: A conjuncture of four forces. Cities, v. 47, p. 95-106, 2015. Disponível em: <https://www.researchgate.net/profile/Margarita_Angelidou2/publication/277026227_Smart_cities_A_conjuncture_of_ four_forces/links/59f19369aca272cdc7ce1b62/ Smart-cities-A-conjuncture-of-four-forces.pdf $>$. Acesso em: 11 mai. 2018.

ANTHOPOULOS, Leonidas.G.; VAKALI, Athena. Urban planning and smart cities: Interrelations and reciprocities. The future internet assembly: p. 178-189, 2012. Disponível em: <https://link. springer.com/content/pdf/10.1007/978-3-64230241-1_16.pdf>. Acesso em: 11 mai. 2018.

CÂMARA, Samuel Façanha et al. Cidades inteligentes no nordeste brasileiro: análise das dimensões de trajetória e a contribuição da população. Cadernos Gestão Pública e Cidadania, v. 21 , n. 69 , p. 137-157, mai. 2016. Disponível em: <http://bibliotecadigital.fgv.br/ojs/index.php/ cgpc/article/download/57739/61867>. Acesso em: 11 mai. 2018

CARAGLIU, Andrea.; DEL BO, Chiara.; NIJKAMP, Peter. 2011 Smart cities in Europe. Journal of urban technology, v. 18, n. 2, p. 65-82, 2011. Disponível em: <http://dare.ubvu.vu.nl/bitstream/handle/1871/15296/20090048. pdf? sequence=2>. Acesso em: 11 mai. 2018.

CRUZ, Daniel Oliveira et al. Cidades inteligentes: uma visão geral a partir de produções científicas. In: ALVAREZ, Cristina Engel de; BRAGANÇA, Luís, Comunidades urbanas energeticamente eficientes. Vitória, p. 42-53.

DINIZ, Herbertt B. M; SILVA, Emanoel Carlos Gomes Ferraz.; GAMA, Kiev. Uma arquitetura de referência para plataforma de crowdsensing em smart cities. In: IX Brazilian Symposium on Information System, 2015, Goiânia. Disponível em: $<w w w . l b d . d c c . u f m g . b r / c o l e c o e s / s b s i / 2015 / 014$. pdf>. Acesso em: 11 mai. 2018.

ENGINEERING VILLAGE, Compedex and Engineering Index Backfile search fieds. Disponível em: <https://www.engineeringvillage.com/search/quick.url?searchid=cf5db95cM582cM46c7M 8a8dMcdca5b30a0c0\&count $=1$ \&usageOrigin $=\& u$ sageZone=>. Acesso em: 25/07/2017. 
EUROPEAN SMART CITIES, Ranking European

Medium Size Cities 2.0, 2013. Disponível em: <http://www.smart-cities.eu/?cid=3\&ver=2>. Acesso em: 04 mar. 2019.

\section{EUROPEAN SMART CITIES, Ranking European}

Medium Size Cities 3.0, 2014. Disponível em: $<$ http://www.smart-cities.eu/?cid=3\&ver=3>. Acesso em: 05 ago. 2017.

EUROPEAN SMART CITIES, Ranking European Medium Size Cities, 2007. Disponível em: <http://www.smart-cities.eu/ranking.html>. Acesso em: 04 mar. 2019.

GIFFINGER, Rudolf et al. City-ranking of European medium-sized cities. In: 51st IFHP World Congress ("Future of Cities"), Copenhagen, 2007. Disponível em: <www.smartcity-ranking.eu/ download/city_ranking_final.pdf $>$. Acesso em: 11 mai. 2018

GIFFINGER, Rudolf.; GUDRUN, Haindlmaier. Smart cities ranking: an effective instrument for the positioning of the cities?. ACE: Architecture, City and Environment, ano 4,v. 12, p. 7-25, fev. 2010. Disponível em: <https://upcommons. upc.edu/bitstream/handle/2099/8550/ACE_12_ SA_10.pdf>. Acesso em: 11 mai. 2018.

GIFFINGER, Rudolf.; PICHLER-MILANOVIC, Nataša. Smart cities: Ranking of European medium-sized cities, Centre of Regional Science, Vienna UT. 2007.
GUIMARÃES, José Geraldo de Araujo. Cidades inteligentes: proposta de um modelo brasileiro multi-ranking de classificação. Tese (Doutorado em Administração) - Faculdade de Economia, Administração e Contabilidade, Universidade de São Paulo, São Paulo, 2018.

KOMNINOS, Nicos.; SEFERTZI, Elena. Intelligent cities: R\&D offshoring, Web 2.0 product development and globalization of innovation systems.

Second Knowledge Cities Summit, ano 2009, p. 1-8, set. 2009. Disponível em: <www.urenio.org/ komninos/wp-content/uploads/2014/01/2009-Intelligent-Cities-Shenzhen-2009-Komninos-Sefertzi-Paper.pdf $>$. Acesso em: 11 mai. 2018.

LOMBARDI, Patrizia et al. An analytic network model for Smart cities. In: Proceedings of the 11th International Symposium on the AHP, Sorreto, jun. 2011. Disponível em: <www.academia. edu/download/30994628/63_0116_Giordano. pdf $>$. Acesso em: 11 mai. 2018.

LUXEMBOURG, Le Gouvernement du Grand-Duché de Luxembourg. Digital Luxembourg. Disponível em: <http://www.digital-luxembourg. public.lu/en/>. Acesso em: 25/07/2017.

MAZZO, Artur de Lazzari. Governança e análise fenomenológica em smart cities: um estudo da Amsterdam Smart City (ASC). Dissertação (Mestrado em Administração de Organizações) 
- Faculdade de Economia, Administração e Contabilidade, Universidade de São Paulo, Ribeirão Preto. 2018.

NAM, Taewoo.; PARDO, Theresa. A. Conceptualizing smart city with dimensions of technology, people, and institutions. In: Proceedings of the 12th annual international digital government research conference: digital government innovation in challenging times, College Park ACM, 2011. Disponível em: <https://www.ctg.albany. edu/publications/journals/dgo_2011_smartcity/ dgo_2011_smartcity.pdf $>$. Acesso em: 11 mai. 2018.

NATIONS, United. World Urbanization Prospects: The 2014 Revision, Highlights department of economic and social affairs. Population Division, United Nations, v. 1, p. 1-3, 2014.

NEIROTTI, Paolo et al. Current trends in Smart City initiatives: Some stylised facts. Cities, v. 38, p. 25-36, jun. 2014. Disponível em: <https://www.sciencedirect.com/science/article/ pii/S0264275113001935>. Acesso em: 11 mai. 2018.

PRZEYBILOVICZ, Erico et al. A tale of two "smart cities": investigating the echoes of new public management and governance discourses in smart city projects in Brazil. In: 51 st Hawaii International Conference on System Sciences Honolulu, Havaí, EUA: University of Hawaii at Manoa Library, sd, 2018, . Disponível em <https://scholarspace. manoa.hawaii.edu/handle/10125/50202> Acesso em: 01 mar. 2019.

PUPO, Alexandre Silveira. Cidades inteligentes baseadas em tecnologias de informação e comunicação: experiências em regiões urbanas sob a ótica da sustentabilidade. Dissertação (Mestrado em Administração) - Faculdade de Economia, Administração e Contabilidade, Universidade de São Paulo, São Paulo, 2017.

ROCHE, Stéphane et al. Are 'smart cities' smart enough. In: Global geospatial conference, 2012. Disponível em: <http://senseable.mit.edu/papers/pdf/20120513_Roche_etal_SmartCities_ SpatiallyEnabling.pdf>. Acesso em: 11 mai. 2018.

\section{URBAN SYSTEMS. Ranking Connected Smart}

Cities. 2017. Disponível em: <https://www. dropbox.com/s/113h0xk71n8b7qr/Ranking CSC_2017.pdf?dl=0>. Acesso em: 04 ago. 2017

WEISS, Marcos César; BERNARDES, Roberto Carlos; CONSONI, Flávia Luciene. Cidades inteligentes como nova prática para o gerenciamento dos serviços e infraestruturas urbanos: a experiência da cidade de Porto Alegre. Revista Brasileira de Gestão Urbana, v. 7, n. 3, p. 310324, set. 2015. Disponível em: <www.scielo.br/ pdf/urbe/2015nahead/2175-3369-urbe-21753369007003AO01.pdf>. Acesso em: 11 mai. 2018. 